\title{
Investigating Suitable Multiple Access Techniques for Harbor Wireless Communication Systems
}

\author{
Enadevita Fitriyani ${ }^{\mathrm{a}, \mathrm{b}, 1}$, Khoirul Anwar ${ }^{\mathrm{a}, \mathrm{b}, 2}$, Nachwan Mufti Adriansyaha, ${ }^{\mathrm{a}, \mathrm{b}}$, \\ ${ }^{a}$ The University Center of Excellence for Advanced Intelligent Communications (AICOMS), \\ ${ }^{b}$ School of Electrical Engineering, Telkom University, Bandung, 40257, Indonesia \\ Corresponding author: ${ }^{1}$ enadevita@ student.telkomuniversity.ac.id, ${ }^{2}$ anwarkhoirul@telkomuniversity.ac.id
}

\begin{abstract}
The multiple access communications for harbor environment are suffering from both low quality transmission due many metallic obstacles and overloaded networks due to the huge number of users/devices of logistic monitoring. This paper proposes a coded random access (CRA) scheme with a degree distribution specially designed to be suitable to harbor environment to serve many devices and provide communications with high reliability. The degree distribution is optimized using extrinsic information transfer (EXIT) charts by keeping that two EXIT curves do not intersect at low mutual information points. Since we are working on the network level, of which the benefit of broadband communications are directly observed to keep the fairness with the narrowband channel, we convert the gain of broadband multipath Rayleight fading channels into an equivalent gain to the narrowband multipath fading channels based on the Shannon channel capacity and channel coding theory. We then compare CRA to another multiple access scheme, i.e, carrier sense multiple access with collision avoidance (CSMA/CA), because CSMA/CA has better performance than other random access schemes, such as ALOHA and Slotted ALOHA. In this paper, we evaluate the performance of the proposed CRA and CSMA/CA using a series of computer simulation in additive white Gaussian noise (AWGN) and multipath Rayleigh fading channels in terms of throughput and packet-loss rate (PLR). The results confirmed that CRA with degree distribution for harbor communications is more suitable than CSMA/CA as a multiple access technique for harbor wireless communications, because CRA has higher throughput on both AWGN and multipath Rayleigh fading channels with lower PLR. The results of this study are expected to contribute to the development of future harbor communication systems.
\end{abstract}

Keywords- Harbor Communication Systems; Multiple Access; CRA; CSMA/CA; AWGN; Rayleigh Fading.

\section{INTRODUCTION}

Since Indonesia entered the industrial era 4.0, several harbor services are served by Internet of things (IoT), e.g, electronic delivery order (e-DO), auto gate system, e-Billing, e-Payment [1]. This condition causes the increase of devices involved in harbor communications. Study in [2] shows that the active connections of the IoT worldwide from 2015 to 2025 are still increasing. The IoT connected devices are predicted to an amount of 21.5 billion units in 2025 . This number is a number of active nodes/devices or gateways that serve the end-sensors, rather than consumer devices, such as computers and cell phones.

Fig. 1 illustrates a communications scheme, where multiple devices are accessing the relay or base station (BS) in the harbor. The BS decodes only one packet at one timeslot, called a clear packet. If more packets are received at the same time-slot, the collision occurs, then the packets are considered as being lost.

A large number of the IoT devices make the conventional 


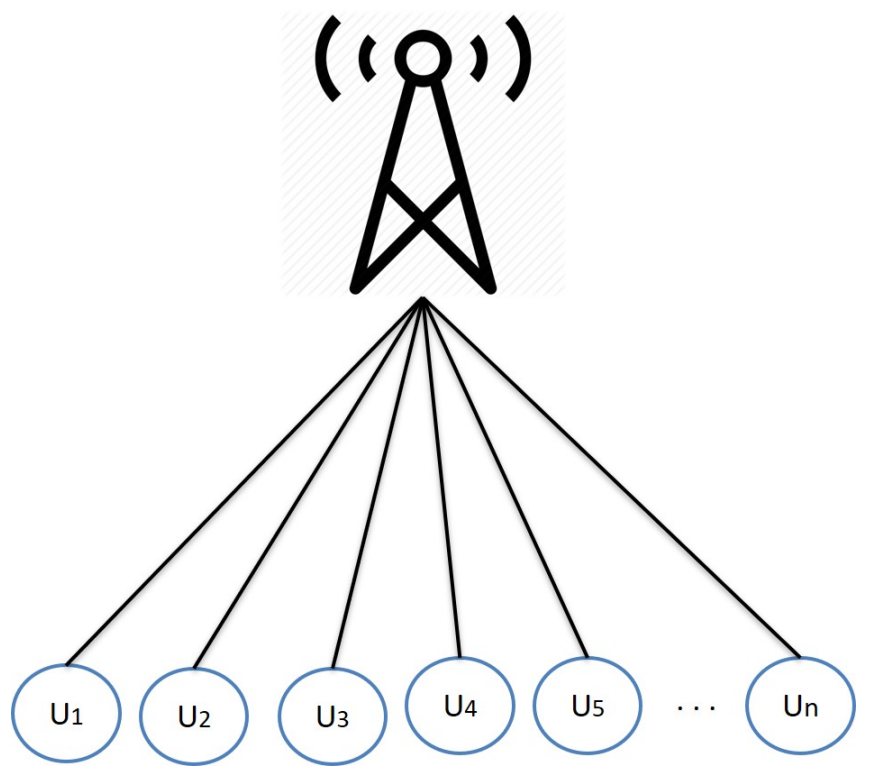

Fig. 1 Structure of massive connectivity network, communications between devices and base station.

multiple access technique is no longer helpful for successful communications because of overloading problem. The conventional multiple access technique, e.g., Time Division Multiple Access (TDMA), Frequency Division Multiple Access (FDMA), and Code Division Multiple Ac- cess (CDMA), do not capability to serve overloaded networks caused by many request/packets from a large number of users or devices. ${ }^{1}$ This problem happens because TDMA, FDMA, and CDMA are orthogonal multiple access technique, and are basically based on a certain scheduling [3], which is, in general, limited. Furthermore, harbor environment is predicted having multipath Rayleigh fading channels due to a large amount of metal. This problem further requires a new multiple access technique that (i) serve a large number of devices and (ii) have good performance although serving large number of users.

A good candidate for the harbor communications is the coded random access (CRA), where the users are allowed to transmits randomly to the relay or BS. The collision is solved using a successive interference cancellation (SIC) technique as used in in low- density parity-check (LDPC) codes to avoid a missing packet because of collision [4], [5]. With CRA, the users are no longer requiring scheduling or dedicated frequency.

The authors [6], [7] use multi-user detection (MUD) scheme to detect higher number of packet simultaneously more than 1 packet/slot. CRA achieves throughput of $T=0.9-3.9$ packet/slot, while another multiple access technique, i.e, ALOHA has throughput of $T=$ $0.184 \mathrm{packet} / \mathrm{slot}$, slotted ALOHA has $T=0.37 \mathrm{packet} / \mathrm{slot}$, and carrier sense multiple access with collision avoidance (CSMA/CA) has $T=0.5-0.8$ packet/slot [8]. The proposed CRA is compared to the CSMA/CA, because

\footnotetext{
${ }^{1}$ In this paper, the terms "user" and "device" are used interchangeably, except specified.
}

CSMA/CA has better performance than that of ALOHA and Slotted ALOHA [9]-[11]. CRA has a better performance than other multiple access techniques, because CRA stores and utilizes collisions to remove interference, while other techniques throw away the collided packets.

In this paper, we propose a new degree distribution for CRA to harbor wireless communication systems. The optimal degree of CRA is designed resistant to fading so that it is expected to be able to minimize the occurrence of broken links. This paper provides analysis with fair comparisons between CRA and CSMA/CA using extrinsic information transfer (EXIT) chart, packet-loss rate (PLR), and throughput performances.

Our contributions are summarized as follows:

1) Multiple access technique is proposed for harbor wireless communications based on the outage probability of the harbor environment channel.

2) This paper compares the performances of CRA and CSMA/CA fairly using the same number of time slots and users.

3) Optimal degree distribution for CRA is proposed to improve the CRA performances in harbor multipath Rayleigh fading channels.

The rest of this paper is organized as follows. Materials and method are presented in Section II. Results and discussion are presented in Section III. Finally, some concluding remarks are presented in Section IV.

\section{MATERIALS AND METHOD}

In this paper, we use the notation of time-slot as slot node (SN) or "square" and user device as user node (UN) or "circle" as indicated in Fig. 2.

\section{A. System Model}

1) $C S M A / C A$ : CSMA/CA minimize collisions probability using collision avoidance algorithm [12]. CSMA/CA implements the "Listen Before Talk", where the sender listens the channel using the carrier sense mechanism to sense a packet from other users. This process is to ensure whether the channel is idle or busy.

Fig. 3 illustrates packet transmission process of CSMA/CA. If the channel is busy, the UN waits until the channel is idle. The length of waiting time is determined based on a random time, called back-off time. The range of random back-off time depends on the number of packet retransmissions, where the time increases exponentially as retransmission is performed. The backoff time ranges from 0 to $2^{c}-1$, where $c$ is the number of collisions experienced by the user. When the channel is in the idle state, the waiting time is ended then UN starts to send the packet [9], [10].

CSMA/CA has a weakness, i.e., the hidden terminal problem when two or more UNs cannot listen to each other [13]. This situation happens because they are outside of the transmission range and are thinking of idle channel. Therefore, they star to send the packets at the same time causing many collisions. 


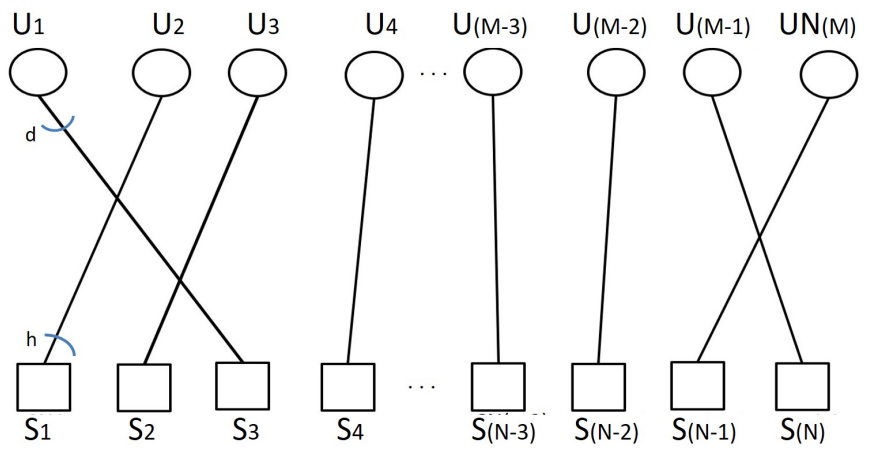

Fig. 2 CSMA/CA mechanism can be represented by a bipartite graph using a degree distribution $\Lambda(x)=x$.

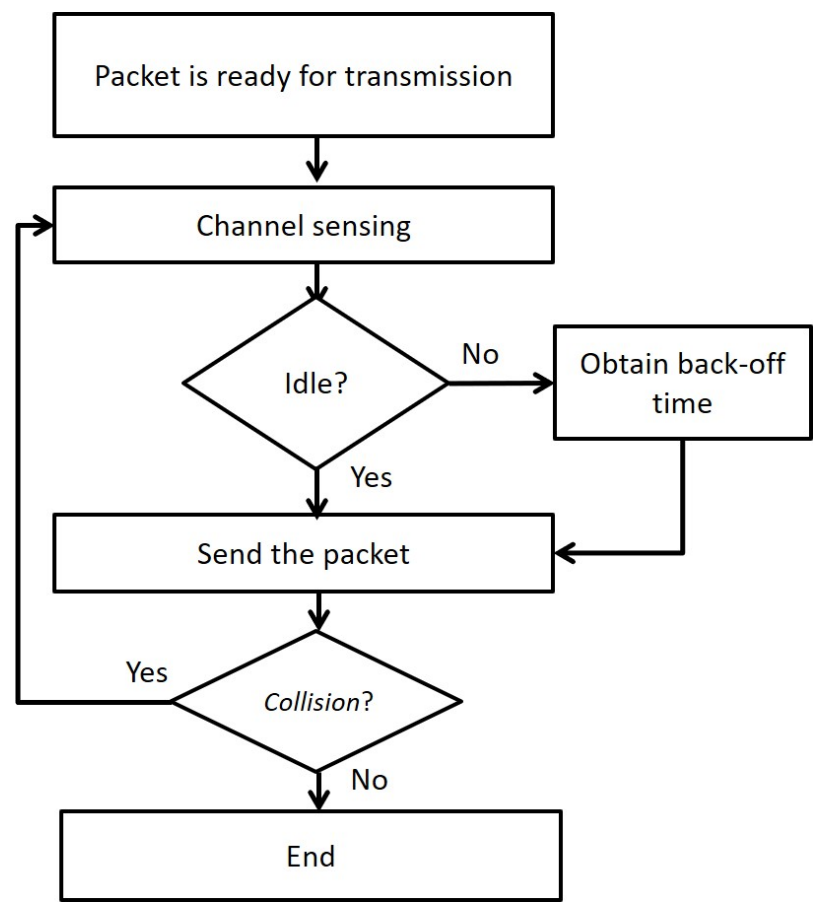

Fig. 3 Working principle of CSMA/CA to avoid collisions.

Fig. 4 shows a hidden terminal problem, where device A can hear device $\mathrm{B}$ and $\mathrm{C}$, but device $\mathrm{C}$ cannot directly hear device B. Similarly, device B cannot hear device C. Since the terminal is hidden, it is in high probability that device $\mathrm{B}$ and device $\mathrm{C}$ are transmitting at the same timeslot resulting in a collision. A collision avoidance scheme can be performed by transmitting a request to send (RTS), clear to send (CTS), and acknowledgement (ACK) [11].

RTS is sent when the device wants to send a packet data, while CTS is a response to the RTS. ACK is a notification that the packet was received by the receiver correctly. When a user sends RTS, CTS, data, and ACK, another device will receive a Network Allocation Vector (NAV). NAV contains information about the time to complete the transmission of a packet. A user that receive an NAV cannot send any packets.

Fig. 5 illustrates a scheme for RTS/CTS, where the RTS should be sent to the receiver before the packet data. After

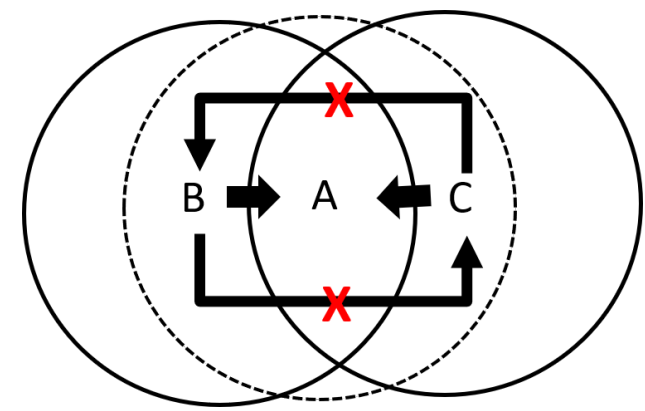

Fig. 4 An illustration of a hidden terminal problem.

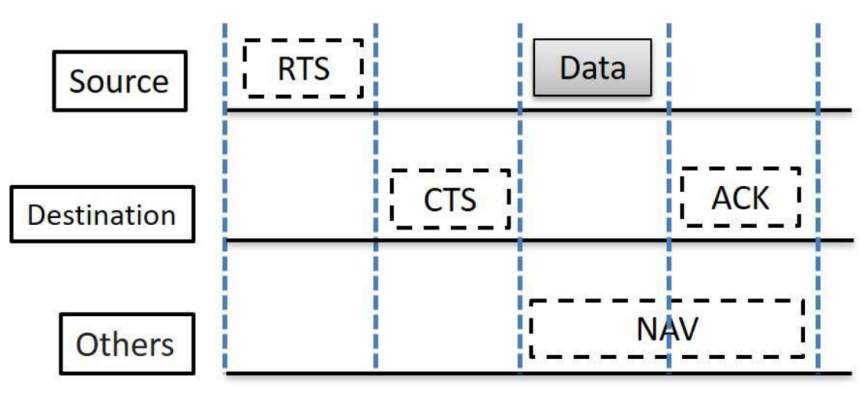

Fig. 5 CSMA/CA with RTS/CTS scheme.

an RTS reached the receiver, the receiver sends a CTS to the sender and broadcasts an NAV to other users. A user that receive an NAV waits until the back-off time is over. A sender that receive a CTS starts to send a packet.

We return back to the details of Fig. 2. Fig. 2 plots a bipartite graph for CSMA/CA connecting SN and UN using a single link. The bipartite graph consists of $M$ users and $N$ time-slot. Each UN only sends one packet using one timeslot, which means that each user only has a degree $d=1$. Based on the description above, the degree distribution of UN in CSMA/CA can be mathematically expressed as

$$
\Lambda(x)=x,
$$

meaning that CSMA/CA has degree $d=1$ as indicated by Fig. 2. If there are two or more packets transmitted, a collision occurs assuming all packets are lost. Since the use of collision avoidance algorithm, the degree of $\mathrm{SN}$ is also one as

$$
\Omega(x)=x .
$$

In this paper, we consider a total of $N=200$ slots and $M=200$ users.

2) The Proposed Multiple Access: In this paper, we propose CRA with optimal degree distribution. We design edge distribution of UN with repetition code to optimize packet transmission. This method can increase throughput, reduce packet-loss rate, and serve large number of users. Fig. 6 shows a bipartite graph of CRA using repetition codes with UN degree distribution

$$
\Lambda(x)=0.5 x^{2}+0.5 x^{4} .
$$




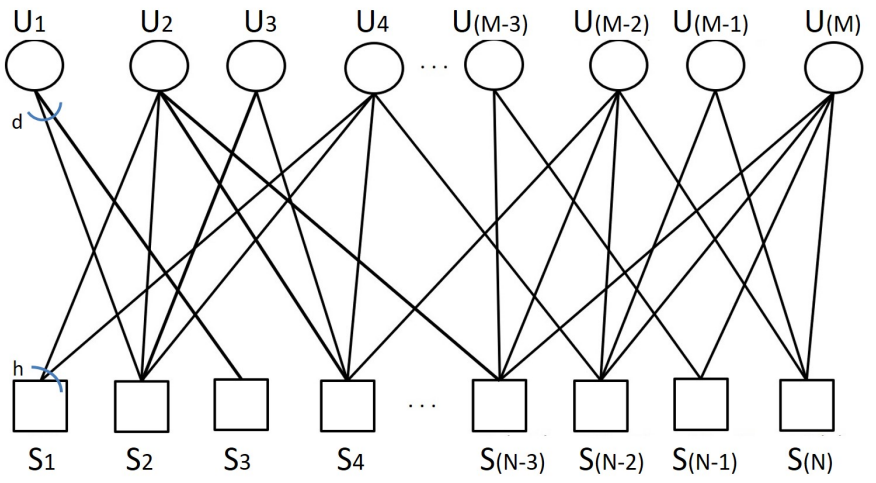

Fig. 6 A bipartite graph of CRA with UN degree distribution $\Lambda(x)=$ $0.5 x^{2}+0.5 x^{4}$

The UN degree distribution in (3) expresses that $50 \%$ UNs send two packets, while other $50 \%$ send four packets at random time slots. In this paper we consider that CRA only decode one user if SN has degree $h=1$, unlike [14] that can decode three users using multi user detection (MUD). The decoding of Fig. 6 is not based on the conventional technique, where the collided packets are discarded. However, the successive interference cancellation (SIC) is performed such that collided packets are still beneficial.

The bipartite graph shown in Fig. 7 represents decoding steps of CRA, where the bipartite graph has user nodes $U=$ $\left\{U_{1}, U_{2}, U_{3}, U_{4}\right\}$ and slot nodes $S=\left\{S_{1}, S_{2}, S_{3}, S_{4}, S_{5}\right\}$. $U$ and $S$, respectively, have message $J$ and receive signal $Y$. The decoding starts by finding user $U$ connected to a slot node $S$ having degree $h=1$. After degree $\mathrm{SN}$ with $h=1$ is found, message of user $U$ is decoded and subtracted from the recived signals $Y$ followed by the removal of the edge connecting the detected user $U$ and all associated slot node $S$. After that, the process is repeated until no more slot nodes having degree $h=1$.

Fig. 7(a) describes an original bipartite graph of CRA with UN degree distribution

$$
\Lambda(x)=0.5 x^{2}+0.5 x^{3} .
$$

$U_{1}$ transmits at $S_{2}$ and $S_{4} ; U_{2}$ transmits at $S_{1}$ and $S_{5} ; U_{3}$ transmits at $S_{2}, S_{3}$ and $S_{4} ; U_{4}$ transmits at $S_{2}, S_{3}$ and $S_{5}$. The received signals become

$$
\begin{aligned}
& Y_{1}=J_{2}, \\
& Y_{2}=J_{1}+J_{3}+J_{4}, \\
& Y_{3}=J_{3}+J_{4}, \\
& Y_{4}=J_{1}+J_{3}, \\
& Y_{5}=J_{2}+J_{4} .
\end{aligned}
$$

Fig. 7(b) shows the first iteration of decoding process in CRA. First of the decoding process is to find an $S N$ having degree $h=1$, i.e, $S_{1}$. The message $J_{2}$ is then detected from the received signal $Y_{1}$. Message $J_{2}$ is then subtracted from $Y_{5}$ to obtain the message $J_{2}$. After the subtraction, the edge of $U_{2}$ connecting to $S_{1}$ and $S_{5}$ are removed from the graph.
The message of $J_{2}$ and $J_{4}$ are then obtained as

$$
J_{2}=Y_{1} .
$$

The second iteration shown in Fig. 7(c) is formed. The process is then repeated as the first iteration to obtain

$$
J_{4}=Y_{5}-J_{2}=Y_{5}-Y_{1} .
$$

The graph of the third iteration is shown in Fig. 7(d), where message of $U_{3}$ is obtained as

$$
J_{3}=Y_{3}-J_{4}=Y_{3}-\left(Y_{5}-Y_{1}\right) .
$$

Finally, we can obtaine message of $U_{1}$ as

$$
\begin{aligned}
J_{1} & =Y_{2}-J_{4}-J_{3} . \\
& =Y_{2}-\left(Y_{5}-Y_{1}\right)-\left(Y_{3}-\left(Y_{5}-Y_{1}\right)\right), \\
J_{1} & =Y_{4}-J_{3}=Y_{4}-\left(Y_{3}-\left(Y_{5}-Y_{1}\right)\right) .
\end{aligned}
$$

Fig. 7(e) shows that all messages have been detected. The decoding process is then stopped, since there no more SN with degree $h=1$ is remaining.

If CSMA/CA or conventional multiple access have bipartite graph original in Fig. 7(a), the successful user is only $U_{2}$, while other users failed. Its because only $S_{1}$ receive one message from $U_{2}$, while other user occurs interference.

3) Degree Distribution: Degree distribution consists of fractions and degree. Fraction is a decimal number that represents the percentage of nodes transmitted using a certain degree. Degree is the number of packages sent by the user in each contention period. The degree distribution of $\mathrm{UN}$ is defined by [15]-[17]

$$
\begin{aligned}
\Lambda(x) & =\sum_{d} \Lambda_{d} x^{d}, \\
\lambda(x) & =\frac{\Lambda^{\prime}(x)}{\Lambda^{\prime}(1)},
\end{aligned}
$$

with $\Lambda(x)$ and $\lambda(x)$ are node-perspective and edgeperspective, respectively. The degree distribution $\mathrm{SN}$ is defined by

$$
\begin{aligned}
\Omega(x) & =\sum_{h} \Omega_{h} x^{h}, \\
\omega(x) & =\frac{\Omega^{\prime}(x)}{\Omega^{\prime}(1)},
\end{aligned}
$$

for node-perspective and edge-perspective, respectively. The erasure probability $\mathrm{SN}$ is defined by $p$ and the erasure probability $\mathrm{UN}$ is $q$, are expressed as

$$
\begin{aligned}
p & =1-\exp \left\{-q \frac{G}{\mathcal{R}}\right\}, \\
q & =\lambda(p), \\
1-p & =\rho(1-q),
\end{aligned}
$$

where $G$ is offered traffic defined as

$$
G=\frac{M}{N}
$$




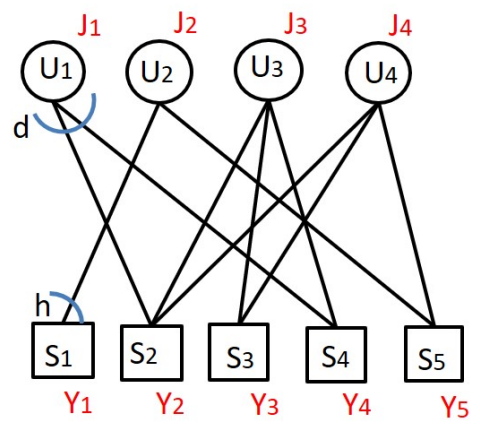

(a) Original.

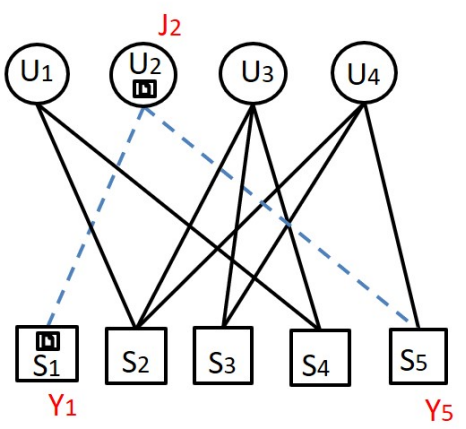

(b) First iteration.

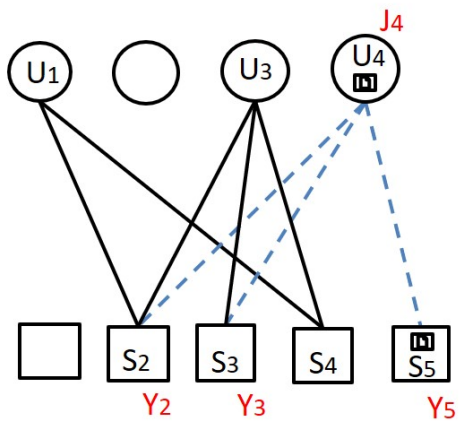

(c) Second iteration.

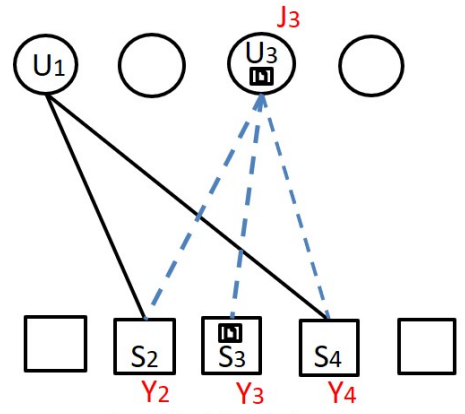

(d) Third iteration.

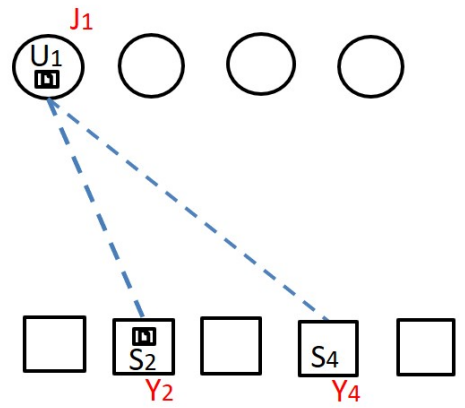

(e) Fourth iteration.

Fig. 7 Bipartite graph for CRA during the decoding process.

with $M$ is the number of users and $N$ is the number of time slots.

$$
\mathcal{R}=\frac{1}{\Lambda^{\prime}(1)},
$$

where $\mathcal{R}$ is network coding rate. ${ }^{2}$

In [15], [18] mutual information of $\mathrm{SN}$ and $\mathrm{UN}$ is

$$
I_{E, S N}=1-p
$$

and

$$
\begin{aligned}
& I_{E, U N}=1-\lambda(q), \\
& I_{E, U N}=1-\lambda\left(1-I_{A, U N}\right),
\end{aligned}
$$

where $I_{A, U N}$ is priori mutual information from UN.

4) Induced Distributions: When the user is moving, the channel is assumed to be following the Rayleigh distribution with probability [19]

$$
\operatorname{Pr}(\gamma)=\frac{1}{\Gamma_{k}} \exp \left(-\frac{\gamma}{\Gamma_{k}}\right)
$$

where $\Gamma_{k}=E\left[|k|^{2}\right]$ is the average received power below power $\gamma^{3}$ In the bipartite graph, the edges connecting users and BTS nodes may be disconnected. We assume that the disconnection can happen when the receive power is below that the threshold $P_{T}$. The probability of signal under

\footnotetext{
${ }^{2}$ The readers are expected to be able distinguish the channel coding rate and network coding rate. In this paper, the channel coding rate is out of the scope of the research.

${ }^{3}$ Please note that (28) in [19] contains errors that should be $P_{r}(\gamma)=$ $\frac{1}{\Gamma} \exp \left(-\frac{\gamma}{\Gamma}\right)$.
}

multipath Rayleigh fading channels having power below a threshold $P_{T}$ is defined as

$$
\operatorname{Pr}\left(\gamma \leq P_{T}\right)=\int_{0}^{P_{T}} \operatorname{Pr}(\gamma) d \gamma=1-\exp \left(-\frac{P_{T}}{\Gamma}\right)
$$

This situation causes the degree distribution changed from original $\Lambda(x)$ to $\bar{\Lambda}(x)$. The induced probability of a user with degree $h$ becomes $\tilde{h}$, at $0 \leq \tilde{h} \leq h$ is

$$
P_{h} \rightarrow \tilde{h}=\left(\begin{array}{c}
\stackrel{h}{\sim} \\
h \stackrel{h}{\sim}
\end{array}\right)\left(P_{d}\right)^{h-\tilde{h}}\left(1-P_{d}\right)^{\tilde{h}},
$$

where $P_{d}$ is probability of an edge dropped as defined by (29). Induced distribution can be defined as

$$
\tilde{\Lambda}(x)=\sum_{\tilde{h}=0}^{H}\left(\sum_{h=0}^{H} \lambda_{h} P_{h \rightarrow \tilde{h}}\right) x^{\tilde{h}},
$$

where $H$ is the maximum degree UN. The (31) can only used to find the induced degree distribution on a single path.

5) Stopping Set: Stopping set is a condition when the received packet cannot be decoded because there are no more degree $h=1$. Based on [20], there are six most important stopping sets with probability, because not all stopping sets have big contribution. In this paper we consider stopping set for one relay with the occurrent probability of stoping set is

$$
\dot{\rho}(\mathcal{S S} i)=P_{O}\left(\mathcal{S S}_{i}\right),
$$

where $i \in\{1.2 \ldots 8\}$ and $P_{O}\left(\mathcal{S}_{i}\right)$ is the occurrence probability of stopping set in one graph [21]. The PLR theory 
calculate by

$$
\begin{aligned}
\dot{P}_{L} \approx & \frac{2 \dot{\rho}\left(S S_{1}\right)+3 \dot{\rho}\left(S S_{2}\right)+4 \dot{\rho}\left(S S_{3}\right)}{M \Lambda_{2}} \\
& +\frac{2 \dot{\rho}\left(S S_{4}\right)+\dot{\rho}\left(S S_{5}\right)+3 \dot{\rho}\left(S S_{6}\right)}{M \Lambda_{2}} \\
& +\frac{\dot{\rho}\left(S S_{4}\right)+2 \dot{\rho}\left(S S_{5}\right)+\dot{\rho}\left(S S_{6}\right)}{M \Lambda_{3}}
\end{aligned}
$$

where $\Lambda_{2}$ is the fraction of degree $l=2$. For example, with $\Lambda(x)=0.8 x^{2}+0.2 x^{4}, \Lambda_{2}=0.8$.

6) Optimal Degree: Optimal degree is a degree of CRA that has highest throughput and lowest PLR. The optimal degree of CRA for harbor channel is obtained by EXIT chart, which induced degree distribution that considers channel with equivalent gain $g_{m}$. The gain $g_{m}$ is the gain given to the single-path channel $h$ as equivalent of the gain obtained due to multipath Rayleigh fading channels between the sensors and relay station. Gain $g_{m}$ is obtained by assuming the gain that make the outage probability of a single path drop to multipath at a specific SNR that targeted for device operation. To get the ideal gain $g_{m}$ is use outage probability based on the Shannon limit for each $h$ channel.

Based on the Shannon channel capacity, the gain $g_{m}$ is obtained by performing a simulation to satisfy

$$
P\left(\log _{2}\left(1+\frac{|h|^{2} \cdot g_{m}}{2 \sigma^{2}}\right)>\mathcal{R}\right)=P_{\text {out }}^{\mathfrak{m}},
$$

where $\sigma^{2}$ is the noise variance for the specified SNR, i.e. $13 \mathrm{~dB}, \mathcal{R}$ is the physical layer channel coding rate. In this paper, we consider no channel coding for simplicity in analysis, therefore, the coding rate $\mathcal{R}=1$. $P_{\text {out }}^{\mathfrak{m}}$ is the harbor channel outage probability of multipath fading which is $P_{\text {out }}^{\mathfrak{m}}=0.04934$ as shown in Fig. 8. The Fig. 8 also shows the outage probability at SNR $\gamma=20 \mathrm{~dB}$ that gives $P_{\text {out }}^{\mathfrak{m}}=0.00143$.

This paper performs a simulation using (34) with 10,000 channel realizations $k$ and finds gain $g_{m}=0.5$ for $\gamma=$ $13 \mathrm{~dB}$ and $g_{m}=4.5$ for $\gamma=20 \mathrm{~dB}$. The value of $g_{m}=4.5$ is a great value at $\gamma=20 \mathrm{~dB}$, then there is no broken link that optimal degree distribution equals to $\Lambda_{o}(x)=0.5 x^{2}+$ $0.5 x^{4}$ which is the optimal degree distribution of the AWGN channel.

If the monitoring device is required to run at $\gamma<20 \mathrm{~dB}$, then the optimal degree of distribution must be searched again. This paper searches for the optimal degree at $\gamma=$ $13 \mathrm{~dB}$ using $g_{m}=0.5$ as shown in Fig. 9. The optimal degree distribution is search through the EXIT chart based on the induced degree distribution which has been derived based on $g_{m}=0.5$ and the network rate at (24).

The results of the degree distribution to be evaluated are shown in Table 1. The empty part shows the degree distribution that produces bad performance in multipath Rayleigh fading channels, while the part that contains the degree distribution shows that it has a good performance. From some good degree distributions, this paper chooses $\Lambda_{p}(x)=0.2 x^{2}+0.8 x^{4}$ as the optimal degree distribution.

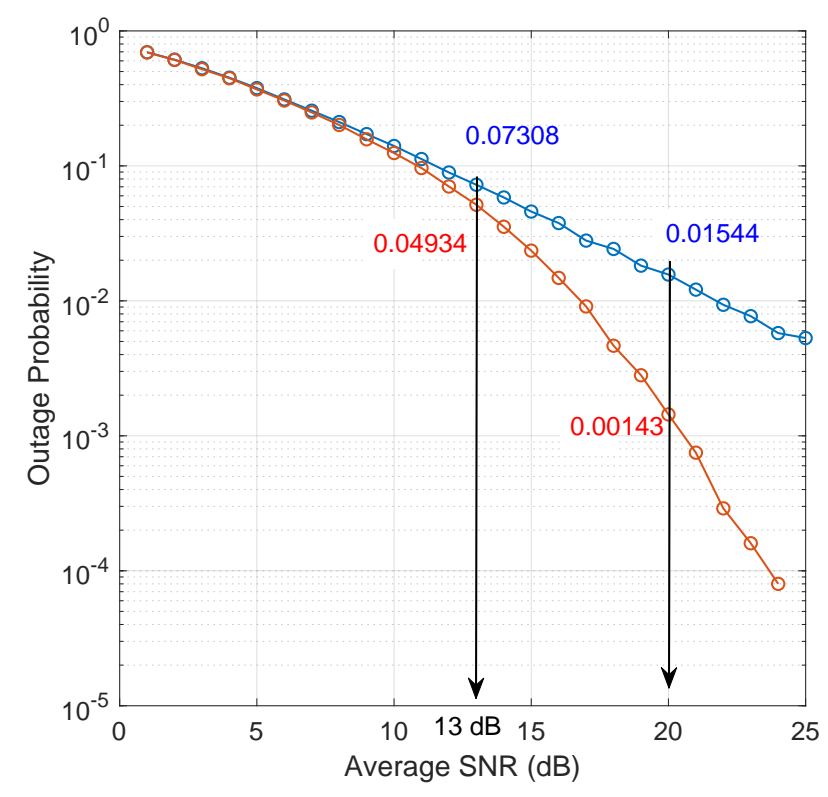

Fig. 8 The results of the harbor-channel performance outage with QPSK modulation and $R=1$.

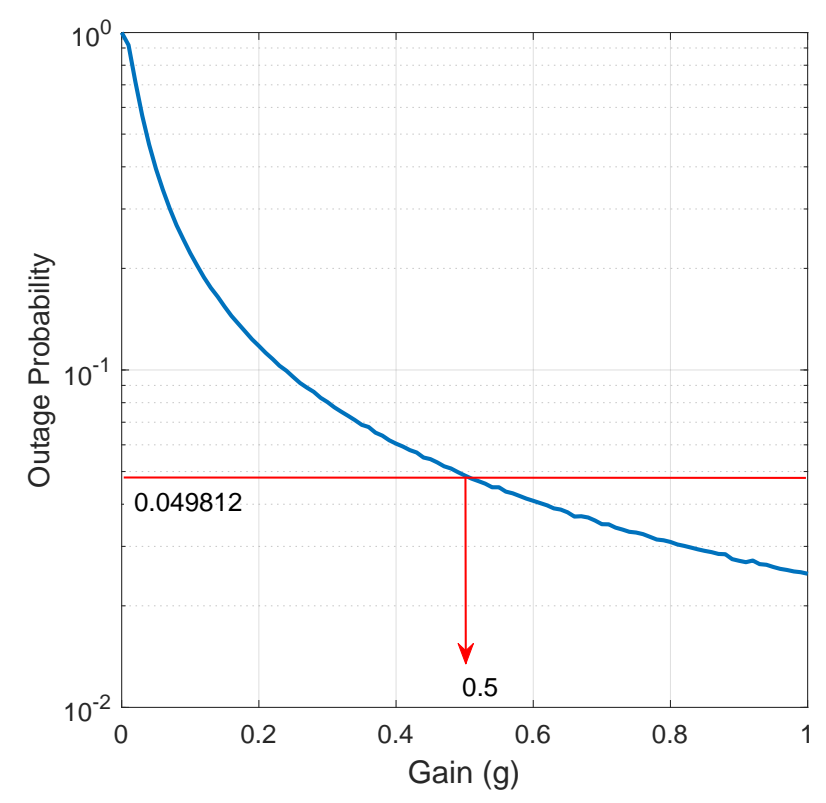

Fig. 9 Equivalent outage performace to gain at SNR $13 \mathrm{~dB}$.

\section{B. AWGN Channel}

AWGN is a channel that does not depend on multipath, fading, frequency selectivity, interference, and dispersion. The resulting noise is additive, white, and sample noise based on the Gaussian distribution.

Additive means that noise is added to the signal while white means that the noise does not depend on the operating system frequency and has a constant power density. The signal that passes through the AWGN channel is modeled 
TABLE I

THE RESULTS OF THE DEGREE COMPARISON.

\begin{tabular}{|c|c|c|c|c|c|c|c|c|c|c|}
\hline Ratio & $x^{2}, x^{3}$ & $x^{2}, x^{4}$ & $x^{2}, x^{5}$ & $x^{2}, x^{6}$ & $x^{3}, x^{4}$ & $x^{3}, x^{5}$ & $x^{3}, x^{6}$ & $x^{4}, x^{5}$ & $x^{4}, x^{6}$ & $x^{5}, x^{6}$ \\
\hline $1: 9$ & $\begin{array}{l}x=0.03 \\
x^{2}=0.32 \\
x^{3}=0.65\end{array}$ & $\begin{array}{l}x=0.03 \\
x^{2}=0.13 \\
x^{3}=0.26 \\
x^{4}=0.58\end{array}$ & - & - & - & - & - & - & - & - \\
\hline $2: 8$ & $\begin{array}{l}x=0.04 \\
x^{2}=0.36 \\
x^{3}=0.6\end{array}$ & $\begin{array}{l}x=0.04 \\
x^{2}=0.2 \\
x^{3}=0.22 \\
x^{4}=0.54\end{array}$ & - & - & - & - & - & - & - & - \\
\hline $3: 7$ & $\begin{array}{l}x=0.07 \\
x^{2}=0.39 \\
x^{3}=0.54\end{array}$ & $\begin{array}{l}x=0.05 \\
x^{2}=0.27 \\
x^{3}=0.18 \\
x^{4}=0.5\end{array}$ & $\begin{array}{l}x=0.05 \\
x^{2}=0.25 \\
x^{3}=0.04 \\
x^{4}=0.23 \\
x^{5}=0.43\end{array}$ & - & $\begin{array}{l}x=0.01 \\
x^{2}=0.1 \\
x^{3}=0.42 \\
x^{4}=0.47\end{array}$ & - & - & - & - & - \\
\hline $4: 6$ & - & - & - & $\begin{array}{l}x=0.09 \\
x^{2}=0.32 \\
x^{3}=0.01 \\
x^{4}=0.04 \\
x^{5}=0.19 \\
x^{6}=0.35\end{array}$ & - & - & - & - & - & - \\
\hline $5: 5$ & - & - & - & - & - & - & $\begin{array}{l}- \\
x=0.01\end{array}$ & - & - & - \\
\hline $6: 4$ & - & - & - & - & - & $\begin{array}{l}x=0.13 \\
x^{2}=0.51 \\
x^{3}=0.1 \\
x^{4}=0.26\end{array}$ & $\begin{array}{l}x^{2}=0.14 \\
x^{3}=0.47 \\
x^{4}=0.04 \\
x^{5}=0.12 \\
x^{6}=0.23\end{array}$ & - & - & - \\
\hline $7: 3$ & - & - & - & - & - & - & - & - & - & - \\
\hline $8: 2$ & - & - & - & - & - & - & - & - & - & - \\
\hline $9: 1$ & - & - & - & - & - & - & - & - & - & - \\
\hline
\end{tabular}

with

$$
\mathbf{y}=k \cdot \mathbf{x}+\mathbf{n},
$$

where $k=1, \mathbf{x}$ is the symbol, and $\mathbf{n}$ is the noise vector.

\section{Multipath Rayleigh Fading Channels}

In the wireless communication systems at the harbor, the signal from the transmitter spreads in all directions, resulting in reflected, diffracted, scattered, and direct waves. This condition causes the path length, phase, and arrival time of each wave to be different at the receiver. Multipath occurs when the signal of the receiver changes from time to time [12].

The received signal under multipath Rayleigh fading is defined as

$$
\begin{aligned}
\mathbf{y} & =\mathbf{K} \cdot \mathbf{x}+\mathbf{n} \\
& \approx g_{m} \cdot \mathbf{x}+\mathbf{n}
\end{aligned}
$$

where $g_{m}$ is the channel gain in (34).
Rayleigh fading distribution describes the variable timing behavior of the receiver of the multipath signal. The equation for the Rayleigh distribution is

$$
P(y)=\frac{y}{\Gamma} \exp \left(-\frac{y^{2}}{2 \Gamma}\right),
$$

where $\Gamma=E\left[y_{I}^{2}\right]=E\left[y_{Q}^{2}\right]$ is the expected average power of the received signals with $y_{I}$ and $y_{Q}$ being the real and imaginary parts of the received signals $y$, respectively.

Signals are then passing through the multipath Rayleigh fading channels as defined in (35) with $k$ being a random complex.

\section{EXIT Analysis}

EXIT charts are used to analyze and design iterations in the decoder system. There are two things that are contained in a good EXIT chart, i.e., (a) it does not cross before reaching the point $(1,1)$ and (b) has a small or narrow gap. 
The analysis is carried out by considering priori mutual information as input $I_{A}$, aposteriory $I_{P}$ as the value obtained from the decoding process, and extrinsic mutual information $I_{E}$ as output.

This paper compares CSMA/CA and CRA fairly in order to know their characteristics and efficiency. This paper uses BEC channel for EXIT analysis based on measurement of mutual information as

$$
I=1-v,
$$

$v=\{p, q\}$. EXIT chart is constructed from the UN degree distribution edge-perspective $\lambda(x)$ that is owned by each system. The $\lambda(x)$ is obtained from UN degree distribution node's perspective $(\Lambda(x)$ following (16). The EXIT chart is plotted in the form of an extrinsic mutual information obtained from (25)-(27). Extrinsic mutual information SN of CSMA/CA is simulated to approximate the maximum throughput, while a priori mutual information UN on CRA for original degree is obtained from

$$
\begin{aligned}
\Lambda_{o}(x) & =0.5 x^{2}+0.5 x^{4}, \\
\lambda_{o}(x) & =0.33 x+0.67 x^{3} .
\end{aligned}
$$

The proposed degree is

$$
\begin{aligned}
\Lambda_{p}(x) & =0.2 x^{2}+0.8 x^{4}, \\
\lambda_{p}(x) & =0.11 x+0.89 x^{3} .
\end{aligned}
$$

In fading channels, degree $\Lambda_{o}(x)$ and $\Lambda_{p}(x)$ are

$$
\begin{aligned}
& \tilde{\Lambda}_{o}(x)=0.1 x+0.43 x^{2}+0.15 x^{3}+0.32 x^{4}, \\
& \tilde{\Lambda}_{p}(x)=0.04 x+0.2 x^{2}+0.23 x^{3}+0.54 x^{4},
\end{aligned}
$$

because some link are disconnected and have a priori mutual information obtained from

$$
\begin{aligned}
& \tilde{\lambda}_{o}(x)=0.037+0.32 x+0.17 x^{2}+0.48 x^{3}, \\
& \tilde{\lambda}_{p}(x)=0.01+0.12 x+0.21 x^{2}+0.66 x^{3} .
\end{aligned}
$$

following (26) and (27).

This paper evaluates EXIT chart in AWGN and multipath Rayleigh fading channels. In AWGN channel, we use rate $\mathcal{R}=0.33$ for CRA and $\mathcal{R}=1$ for CSMA/CA.

Fig. 10 shows EXIT for CRA with proposed optimal degree distribution $\Lambda_{o}(x)$ at $G=0.868$ and for CSMA/CA in AWGN channel. The non-intersecting EXIT curves of CRA indicate that throughput can achieve at $T=0.868$ packets/slot. ${ }^{4}$ The EXIT curves of CSMA/CA are straight lines because CSMA/CA does not have a random mechanism of transmission and only has degree one. The SN and UN of CSMA/CA curves intersect at point $(0,0.6)$ because they have a maximum throughput $T=0.6$ packet/slot. Fig. 10 shows that CRA has better performance than CSMA/CA in AWGN channel.

Fig. 11 shows the EXIT chart of CRA and CSMA/CA in multipath Rayleigh fading channels at $\gamma=13 \mathrm{~dB}$ with $\mathcal{R}=0.37$ for $\mathrm{CRA} \Lambda_{o}(x), \mathcal{R}=0.47$ for $\mathrm{CRA} \Lambda_{p}(x)$,

\footnotetext{
${ }^{4}$ The throughput in this paper has a unit of packets/slot, while offered traffic $G$ does not.
}

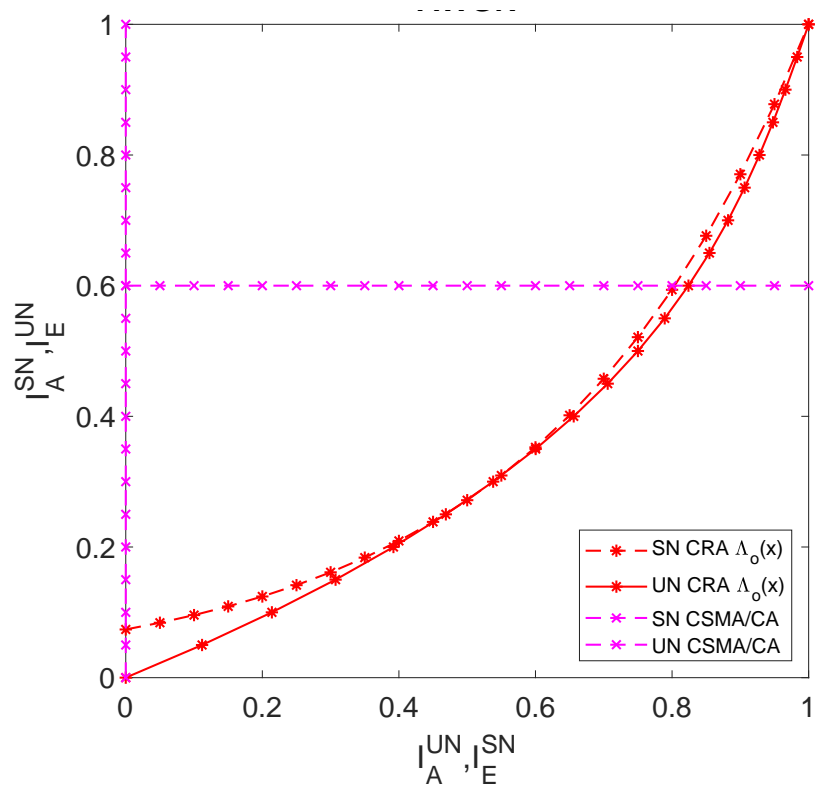

Fig. 10 Comparison of EXIT chart between CRA and CSMA/CA in AWGN channel at $G=0.868$.

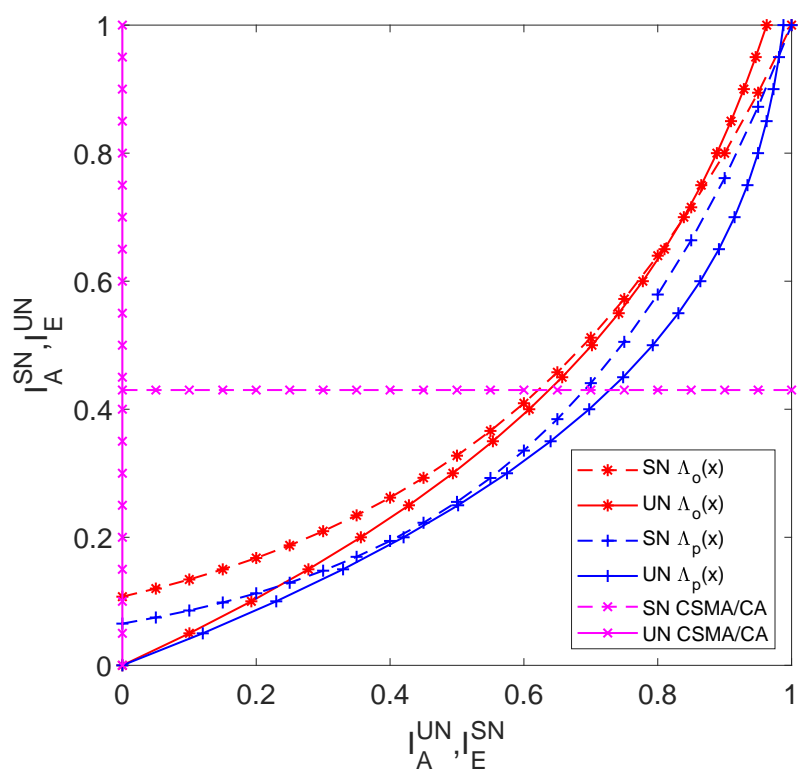

Fig. 11 Comparison of EXIT chart performance between CRA with $\Lambda_{p}(x)$, CRA with $\Lambda_{o}(x)$ and CSMA/CA in multipath Rayleigh fading channels.

and $\mathcal{R}=1$ for CSMA/CA. EXIT chart for SN and UN of $\Lambda_{o}(x)$ is no longer optimal because it intersects at a poin far before $(1,1)$, while EXIT curve of $\Lambda_{p}(x)$ intersects at a point close to $(1,1)$. It indicates that $\Lambda_{p}(x)$ is better than $\Lambda_{o}(x)$. Fig. 11 shows that the CSMA/CA EXIT curve intersect at a point $(0.0 .43)$ showing that the CSMA/CA throughput is at $T=0.43$ packet/slot.

\section{RESUlTS AND DiscusSiON}

\section{A. Packet-Loss Rate}

This paper evaluates the PLR of CRA and CSMA/CA with $M=200$ and $N=200$. Fig. 12 shows the PLR 


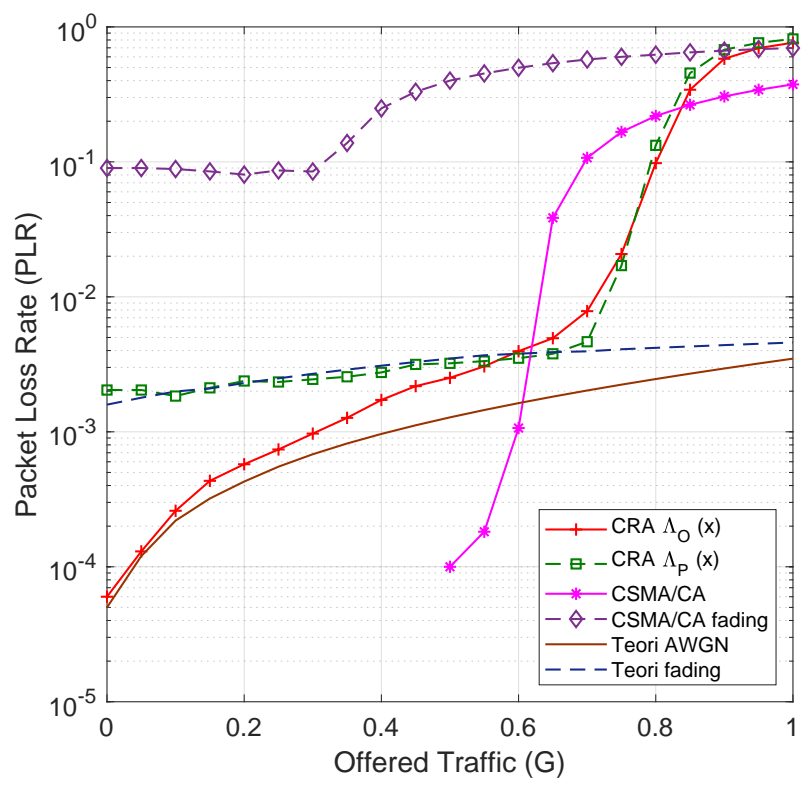

Fig. 12 Comparison of PLR performance between CRA with $\Lambda_{p}(x)$, CRA with $\Lambda_{o}(x)$, and CSMA/CA in multipath Rayleigh fading channels.

curves for CRA and CSMA/CA in AWGN and multipath Rayleigh fading channels. This simulation refers to the packet decoding capability of each system. CSMA/CA and CRA are only able to decode one packet, so when there is a packet that exceeds the system's capability, the other packets are considered as a loss.

PLR is a ratio of the number of failed packet have been sent and the total packets have been sent defined by

$$
P=\frac{c}{T_{p}}
$$

where $c$ is the number of failed packets and $T_{p}$ is the number of packets sent.

Fig. 12 shows PLR performance in AWGN and multipath Rayleigh fading channels for CSMA/CA and CRA with $\Lambda_{p}(x)$ and $\Lambda_{o}(x)$. Theoretical PLR is added to this figure for reference. Theoretical PLR is obtained from (33) [19], [20]. PLR of CRA with $\Lambda_{p}(x)$ and $\Lambda_{o}(x)$ drops at $G=0.7$ with PLR $P_{L}=10^{-2}$ in multipath Rayleigh fading channels and AWGN channels, while CSMA/CA drops at $G=0.63$ in AWGN channel. CSMA/CA has poor performance in multipath Rayleigh fading channels because of the errorfloor occurs at PLR $P_{L}=10^{-1}$ for all offered traffic $G$.

\section{B. Throughput}

Throughput is the ratio of the number of packets that were successfully decoded to the number of packets sent. The throughput is calculated as

$$
T=G(1-P),
$$

where $P$ is PLR and $G$ obtained from (23). This paper uses the PLR (48) to calculate the throughput as shown in Fig. 13.

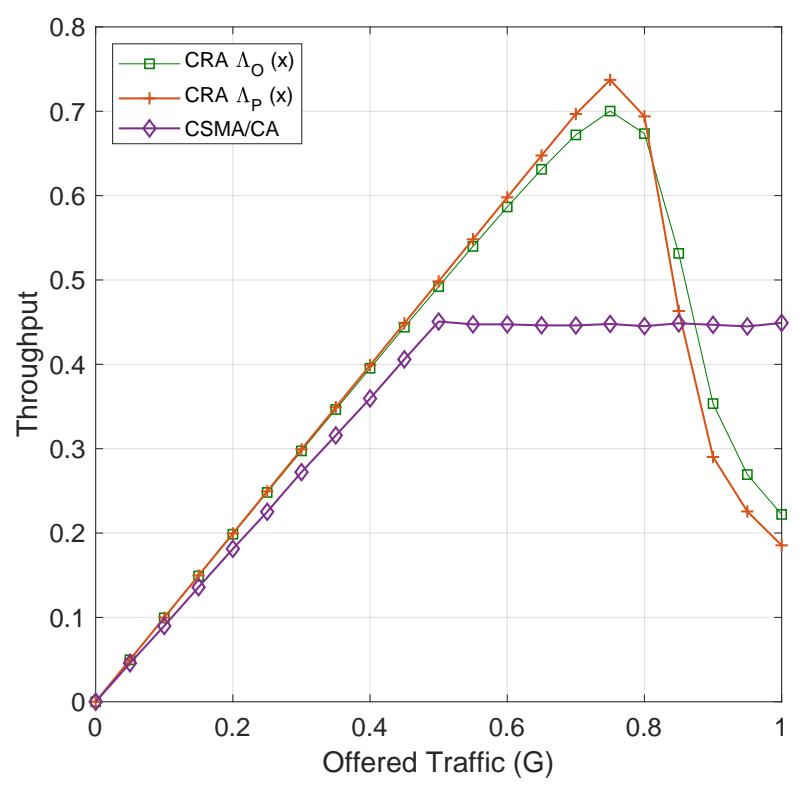

Fig. 13 Comparison of throughput performance between CRA with $\Lambda_{p}(x)$, CRA with $\Lambda_{o}(x)$, and CSMA/CA in multipath Rayleigh fading channels.

Fig. 13 shows throughput in multipath Rayleigh fading channels for CRA with $\Lambda_{p}(x)$ and $\Lambda_{o}(x)$ and CSMA/CA. CRA with $\Lambda_{p}(x)$ has better performances results than CRA with $\Lambda_{o}(x)$ and CSMA/CA. CRA with $\Lambda_{p}(x)$ achieves $T=0.73$ packets/slot, while a CRA with $\Lambda_{o}(x)$ achieves $T=0.7$ packets/slot. This figure shows that CSMA/CA only achieves $T=0.43$ packets/slot in multipath Rayleigh fading channels because of the high error-floor.

\section{CONCLUSION}

This paper has proposed a multiple access technique for harbor communication systems (i) to serve the huge number of users/devices and (ii) to provide low error rate under multipath Rayleigh fading channels. The capability of CRA to serve more users than CSMA/CA has been shown in term of good PLR performances, higher throughput, lower error-floor proven by theoretical performance analyses. We have also derived, by simulation an equivalent channel gain of multipath Rayleigh fading channels based on the outage probability using the Shannon channel capacity and coding theory. This paper has found that $\Lambda_{p}(x)=0.2 x^{2}+0.8 x^{4}$ is an optimal degree distribution for CRA under multipath Rayleigh fading channels and $\Lambda_{o}(x)=0.5 x^{2}+0.5 x^{4}$ is optimal for CRA under AWGN channel. The results show that CRA has better performances than CSMA/CA and is suitable for future IoT networks that involve a huge number of users/devices for harbor wireless communications.

\section{REFERENCES}

[1] D. Andreas, "Pemerintah luncurkan roadmap industri 4.0," April 2018. [Online]. Available: https://tirto.id/ pemerintah-luncurkan-roadmap-industri-40-cHb2 
[2] Statista, "Internet of Things (IoT) active connections worldwide 2015-2025," March 2020. [Online]. Available: https://www.statista. com/statistics/1101442/iot-number-of-connected-devices-worldwide/

[3] O. Shmuel, A. Cohen, and O. Gurewitz, "Performance analysis of opportunistic distributed scheduling in multi-user systems," IEEE Transactions on Communications, vol. 66, no. 10, pp. 4637-4652, 2018.

[4] E. Paolini, C. Stefanovic, G. Liva, and P. Popovski, "Coded random access: applying codes on graphs to design random access protocols," IEEE Communications Magazine, vol. 53, no. 6, pp. 144-150, 2015.

[5] E. Paolini, G. Liva, and M. Chiani, "Coded slotted aloha: A graphbased method for uncoordinated multiple access," IEEE Transactions on Information Theory, vol. 61, pp. 6815-6832, 2015.

[6] A. A. Purwita and K. Anwar, "Vehicular massive multiway relay networks applying graph-based random access," in 2015 IEEE Vehicular Networking Conference (VNC), 2015, pp. 227-234.

[7] - "Massive multiway relay networks applying coded random access," IEEE Transactions on Communications, vol. 64, no. 10, pp. 4134-4146, 2016.

[8] K. Anwar, Juansyah, B. Syihabuddin, and N. M. Adriansyah, "Coded random access with simple header detection for finite length wireless IoT networks," in 2017 Eighth International Workshop on Signal Design and Its Applications in Communications (IWSDA), 2017, pp. 94-98.

[9] A. Mazin, M. Elkourdi, and R. D. Gitlin, "Comparison of Slotted Aloha-NOMA and CSMA/CA for M2M communications in IoT networks," in 2018 IEEE 88th Vehicular Technology Conference (VTC-Fall), 2018, pp. 1-5.

[10] Xiaolong Li and Qing-An Zeng, "Capture effect in the IEEE 802.11 WLANs with rayleigh fading, shadowing, and path loss," in 2006 IEEE International Conference on Wireless and Mobile Computing, Networking and Communications, 2006, pp. 110-115.

[11] Yunli Chen, Qing-An Zeng, and D. P. Agrawal, "Performance analysis and enhancement for IEEE 802.11 MAC protocol," in 10th International Conference on Telecommunications, 2003. ICT 2003., vol. 1, 2003, pp. 860-867 vol.1.
[12] H.Harada and R.Prasad, Sea Transportation Statistics 2018. Artech House, 2002

[13] Yunli Chen, Qing-An Zeng, and D. P. Agrawal, "Analytical modeling of MAC protocol in ad hoc networks," in WIRELESS COMMUNICATIONS AND MOBILE COMPUTING, 2006., 2006, p. 8:45-59.

[14] D. R. Baaqii, Y. Rahayu, and K. Anwar, "Menentukan degree distribution optimal pada coded random access (CRA) dengan multi user detection (MUD) k=3 menggunakan extrinsic information transfer (EXIT) chart," in Jurnal Online Mahasiswa (JOM) Bidang Teknik dan Sains, vol. 6, 2019.

[15] F. M. Pasalbessy and K. Anwar, "Analysis of internet of things (IoT) networks using extrinsic information transfer (EXIT) chart," in 2018 International Seminar on Intelligent Technology and Its Applications (ISITIA), 2018, pp. 281-285.

[16] K. Anwar, "High-dense multiway relay networks exploiting direct links as side information," in 2016 IEEE International Conference on Communications (ICC), 2016, pp. 1-6.

[17] S. Larasati, I. N. A. Ramatryana, and K. Anwar, "High-rate coded random access for non-orthogonal multiple access with human priority," in 2018 2nd International Conference on Telematics and Future Generation Networks (TAFGEN), 2018, pp. 25-30.

[18] A. Ashikhmin, G. Kramer, and S. ten Brink, "Extrinsic information transfer functions: model and erasure channel properties," IEEE Transactions on Information Theory, vol. 50, no. 11, pp. 2657-2673, October 2004.

[19] K. Anwar and R. P. Astuti, "Finite-length analysis for wireless superdense networks exploiting coded random access over rayleigh fading channels," in 2016 IEEE Asia Pacific Conference on Wireless and Mobile (APWiMob), 2016, pp. 7-13.

[20] K. Anwar, "Decoding for wireless super-dense networks and its finite-length analysis for practical applications," in 2016 International Symposium on Electronics and Smart Devices (ISESD), 2016, pp. 347-354.

[21] M. Ivanov, F. Brannstrom, A. G. I. Amat, and P. Popovski, "Error floor analysis of coded slotted aloha over packet erasure channels," IEEE Communications Letters, vol. 19, pp. 419-422, 2015. 